\title{
AN EXPERT SYSTEM FOR NETWORK DESIGN OPERATION AND DIAGNOSIS
}

\author{
نظّم خيير في تصسيم د إدارة ر تطخيص أعطل شبكات الحاسب \\ AIDA O. ABD EL-GAWAD \\ Computer \& Control Dep. , Faculty of Engineering \\ EL-Mansoura University, EL-Mansoura, Egypt .
}

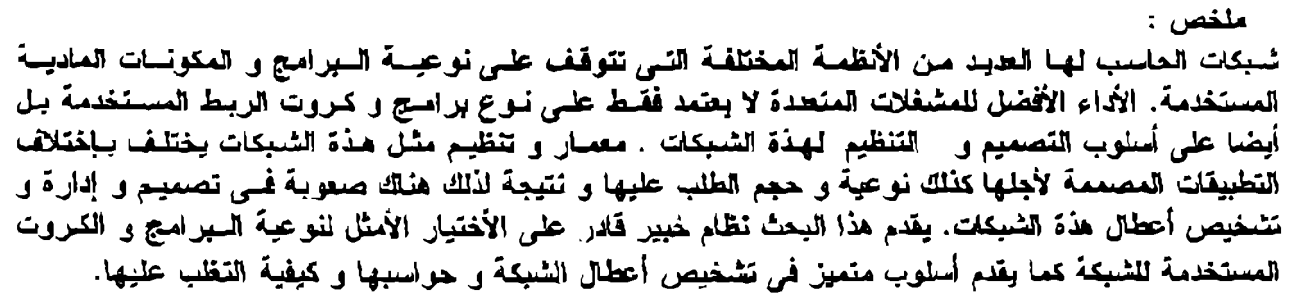

\section{ABSTRACT}

Computer networking has different approaches related to specific software and hardware used. High performance of multiprocessors depends on not only the type of software, and interfacing cards used but also how the network is designed and organized. Architecture and organization of such networks vary according to the application under consideration and the variety of requests and demands required from the network. As a result difficulties are imposed in designing, managing, and diagnosing networks. This paper introduces an expert system that is able to select the proper type and interfacing cards of the network and also, it has an excellent methodology for network fault diagnoses. In addition to, it has a very simple procedure for non expert user for handling any type of networking software.

\section{INTRODUCTION}

Artificial intelligence may be defined as a branch of computer science that is concerned with the automation of intelligent behavior. This implies theoretical principles that include data structures used in knowledge representation, algorithms needed to apply that knowledge in search and inference mechanisms, and both language and programming methodologies used in implementing such techniques. 
Artificial intelligence has many branches such that :-

* Speech recognition,

* Natural language processing ,

* Robotics,

* Computer vision and image processing system

* Expert system :

Expert system is a problem-solving systems that reach expert or at least highly competent levels of perfomance, and that this paper is interested in.

Modem networks use a layered architecture in which each layer is a logical entity that performs certain functions. The services performed by the highest layer are for the network users directly. Each layer provides services for the next layer above it and shields the details of how the services are carried out from this higher layer. Information flow from one level to another depends on the type of interfacing used between each pear of adjacent layers. The parameter setting of such an interface is very important to guarantee accurate and secured data flow. A defined set of different protocols have been introduced each of which defined different set of rules which is necessary for each layer to carry out it's conversation to the corresponding layer at each communication node (1-4). The last few years have witnessed an increasing sophistication in system networking in both hardware and software aspects. Different software packages are now available $[5,6]$ each of which has a different procedure for installation and parameter setting as well as suitable type of network cards. These make the decision of selection network type, parameter setting, and system configuration for both master and servers beside fault system and diagnosis for the different types of networks is a real problem.

In this study an expert system which deals with system networking selection and its hardware and software setting problems is introduced. The system has an excellent methodology for fault diagnoses of network and introduces a very simple procedure for non expert user to define the most suitable network and how to organize and manage. What distinguishes an expert system from a conventional program is not just its expertise, but the way that the expertise is sorted and processed. The proposed system is designed In such a way that make it capable of performing the following:-

(1) Problem solving

(2) Interactive explanations

(3) Knowledge acquisition

Besides, like all software, it is very fast, easy to maintain, and user-friendly. 
Mansoura Engineering Journal (MEJ), Vol.20, No. 2,June $1995 \quad$ E. 73

Expert system design:

Many languages can be used for building expert systems such as PROLOG, OPS5 and LISP, in this work Prolog (PROgramming in LOGic) is used for bullding the control part of the proposed system. Because It is a descriptive language uses facts and rules and its program essentlally consists of a list of logic statements, either in the form of facts or in the form of rules and it can make deduction. The expert system consists of many modules all of them have much graphics, sound and animation, therefore they are designed using $C$ language. The control part of the system defines the type of network to be handle, a certain subject from this type, and which module of this subject is to be executed.

The system is designed in four components, these are :

(1) User interface

(2) Inference engine

(3) knowledge-base

(4) Explanation facilities

this is shown in figure(1-a).

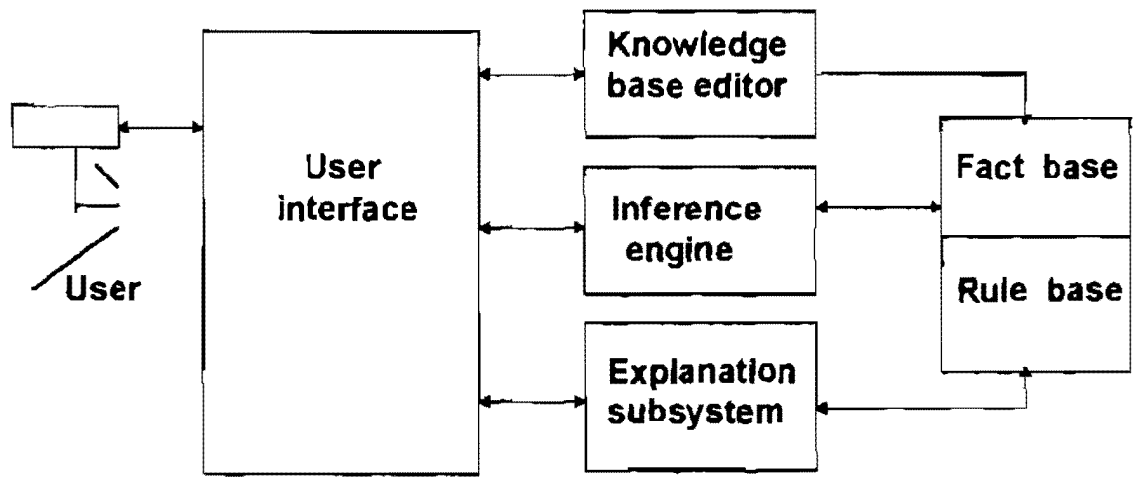

Figure(1-a) Expert system components

A list of a computer program for the module of file server is given in appendix a as a sample module. A list of the prolog program for the control part is listed below.

domains

file=myfile

predicates

goto(symbol,symbol,symbol)

goto1(symbol,symbol)

print_contents(integer)

intro(symbol) 


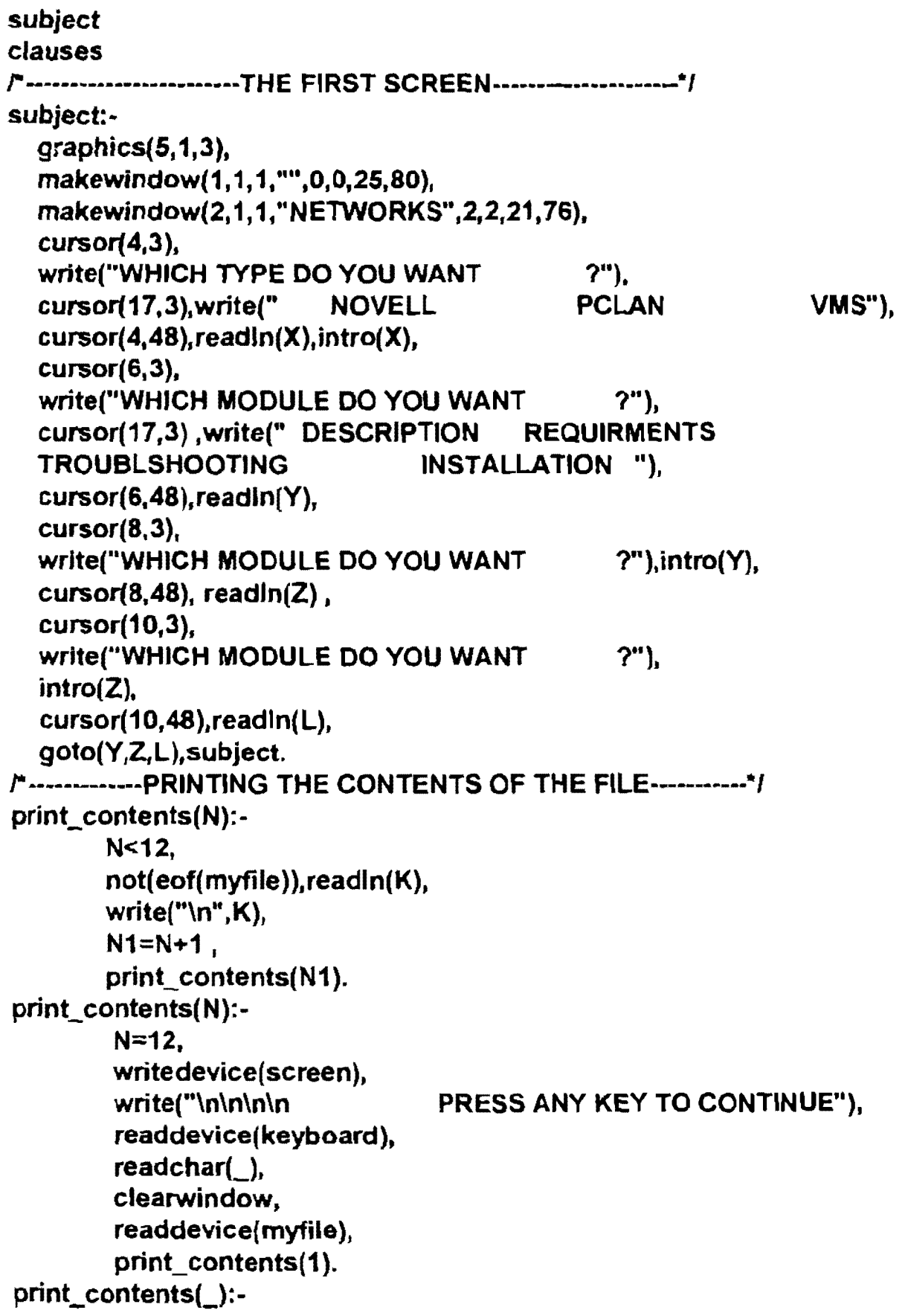




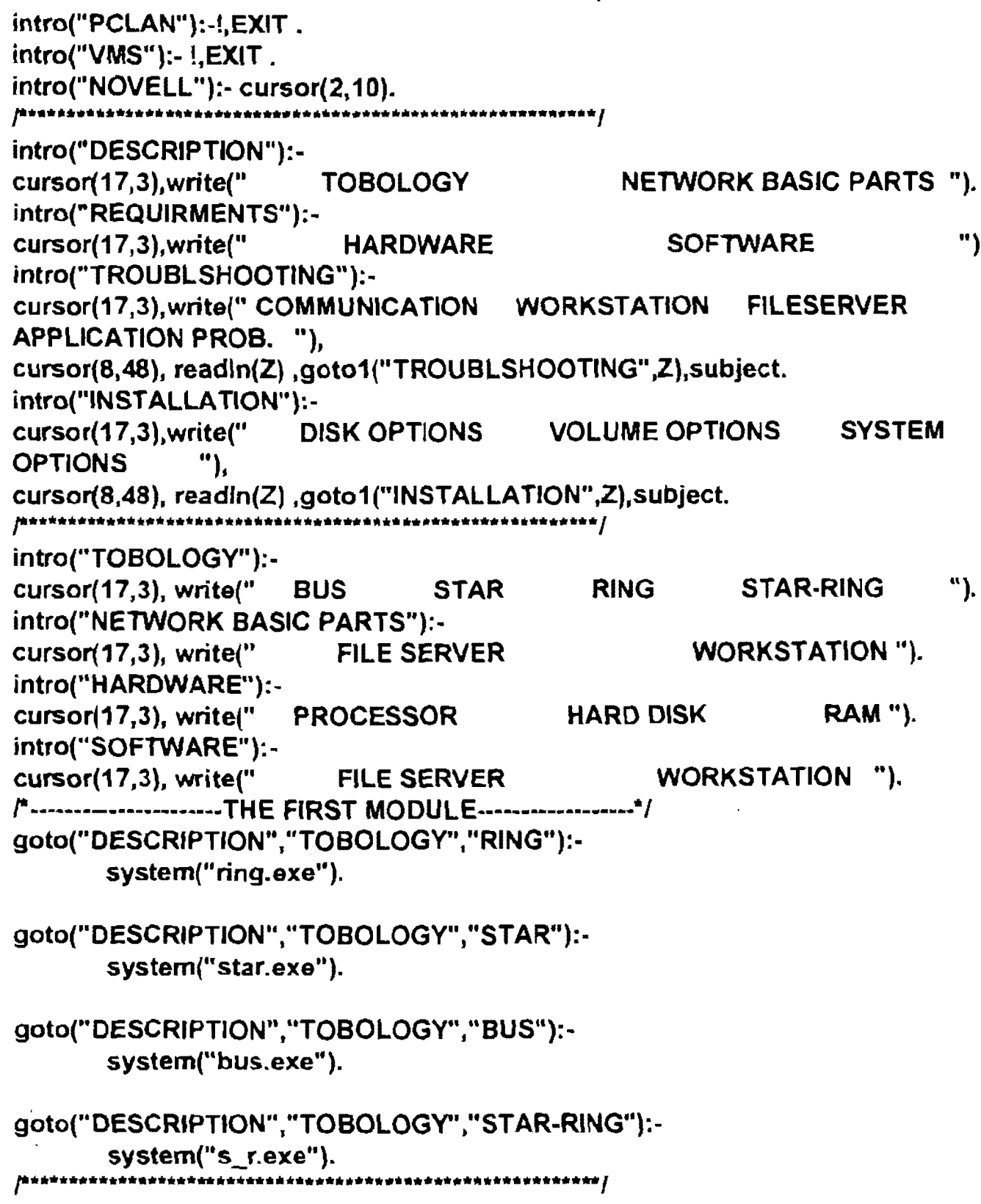


E. 76 DR. AIDA 0. ABD EL-GAWAD

goto("DESCRIPTION","NETWORK BASIC PARTS","FILE SERVER"):openread(myfile,"file_s"), readdevice(myfile), print_contents(1).

goto("DESCRIPTION","NETWORK BASIC PARTS","WORKSTATION"):openread(myfile, "work_s"), readdevice(myfile), print_contents(1).

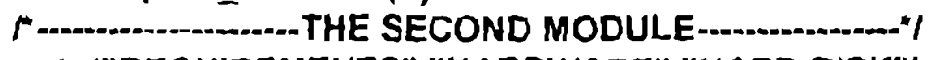

goto("REQUIREMENTS","HARDWARE","HARD DISK"):-

openread(myfile,"hard_d"), readdovice(myfile), print_contents(1) .

goto("REQUIREMENTS","HARDWARE","PROCESSOR"):openread(myfile,"procesor"), readdevice(myfile), print_contents(1).

goto("REQUIREMENTS","HARDWARE","RAM"):openread(myfile,"ram"), readdevice(myfile), print_contents(1).

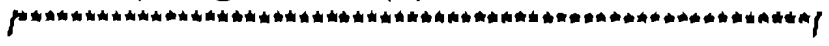

goto("REQUIREMENTS","SOFTWARE","FILE SERVER"):openread(myfile," fille_svr"),readdevice(myfile), print_contents( 1 ).

goto("REQUIREMENTS","SOFTWARE","WORKSTATION"):openread(myfile,"work_st"),readdevice(myfile), print_contents $(1)$.

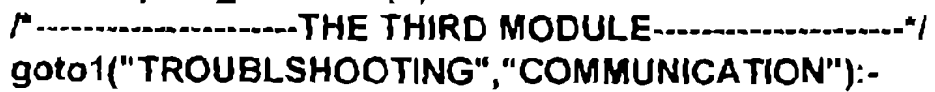
system("communc.exe").

goto1("TROUBLSHOOTING","FILE SERVER"):system("file_v.exe").

goto1("TROUBLSHOOOTING","WORKSTATION"):system("work_st.exe").

goto1("TROUBLSHOOTING","APPLICATION"):system("applic.exe").

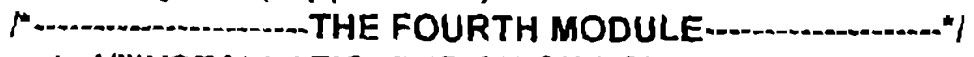

goto1("INSTALLATION","DISK OPTIONS"):system("disk.exe").

goto1("INSTALLATION","VOLUM OPTIONS"):system("volume.exe").

goto1("INSTALLATION","SYSTEM OPTIONS"):system("system.exe").

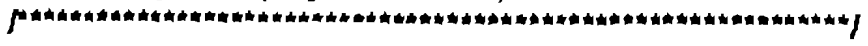

goal

subject. 
User interface:

Many menus are designed for user interfacing. The user interface is the way by which the user communicates with the expert system, and it is built in menus. They are used to select different functions of the system and different parts in each function.A sample of such menus is given in figure(2).

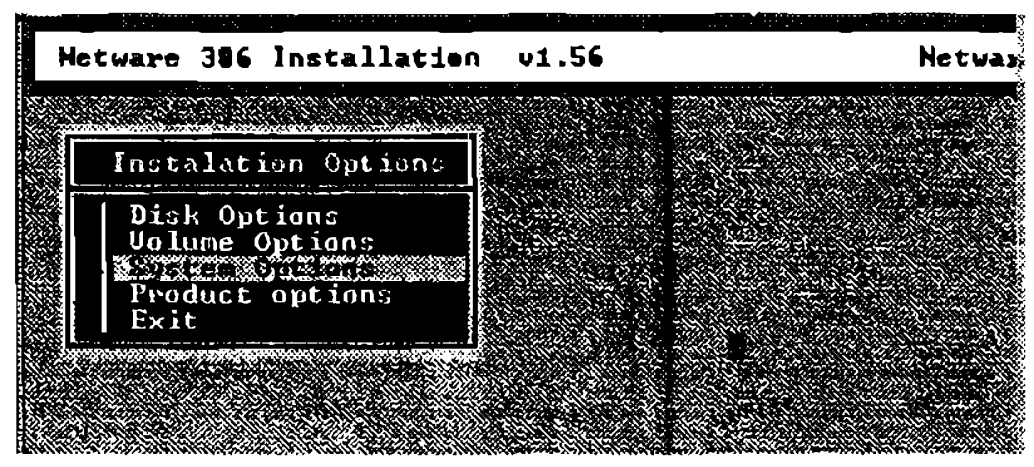

Figure(2) Installation of novell netware 386

\section{Inference engine :}

Inference engine is implemented by different program modules for Description, Requirements, Troubleshooting, Installation. Inference engine is the part of the program that makes the system knows which knowledge-base is required, so it is the linker of the expert system, embodying the main control structures and algorithms. The cycle of the inference engine is designed to select an inference to execute and a data file to manipulate. Using menus you have first to select the type of network NOVELL, PCLAN or VMS, second the required module you want to deal with Description, Installation, Troubleshooting or Requirements after that the system searches about the predicate which satisfies all prevlous requirements and execute it .Figure(3) shows the tree dlagram of the proposed system. Samples of menus are shown in figures(4-6). The Figures show the novell network installation procedure. Methods for interconnecting local area networks are also shown in figure(6). 
E. 78 Dr. AIDA O. ABD EL-GAWAD

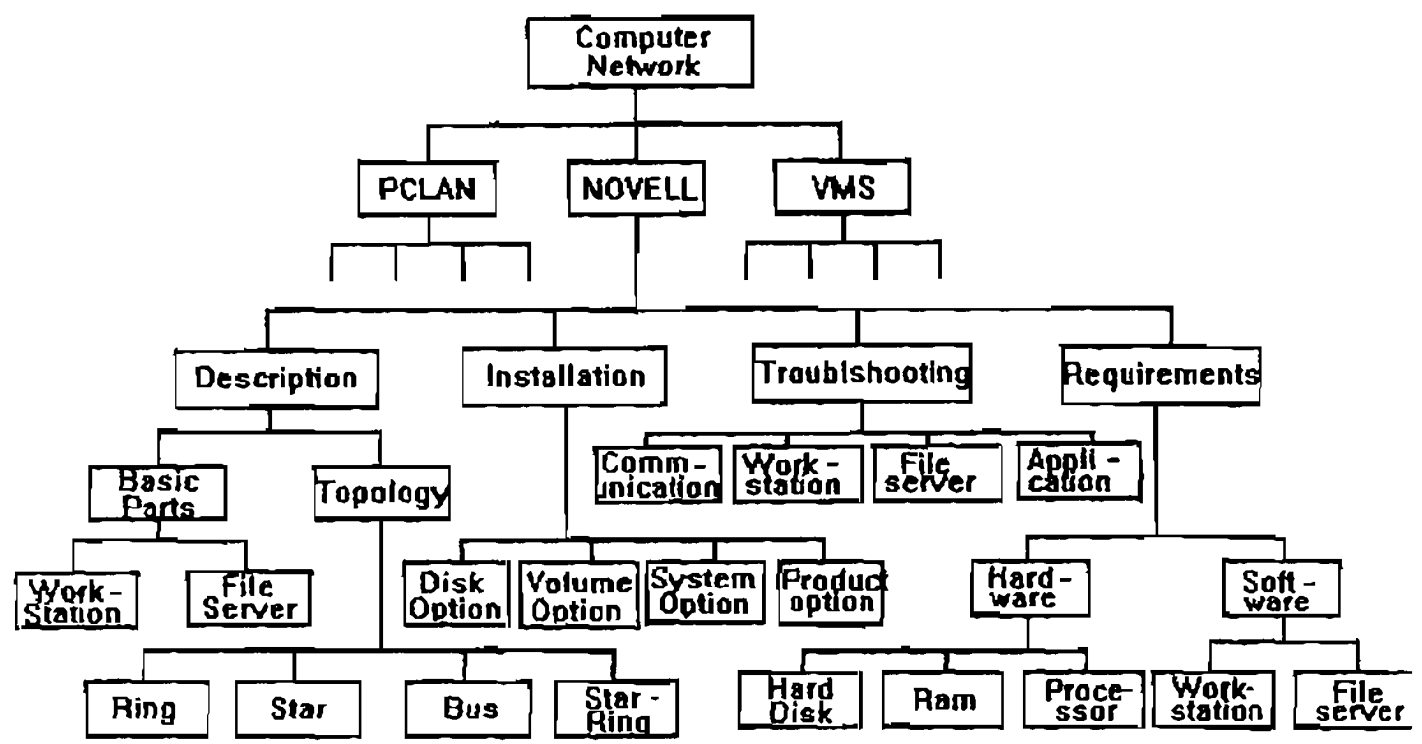

Figure(3) Tree representation of the system

\section{KNOWLEDGE - BASE :}

The knowledge-base comprises a series of rules and facts related to the module which we are dealling with.

\section{Rules:}

It receives the type of the selected network in order to display and define its main function.

EX.

If the function computer network is selected, the predicate :

intro ("COMPUTER NETWORK ") is executed. This predicate is then display the different types of computer network such as Novell, Pclan and Vms, and the user have to select one of them.

If the novell network is chosen, the predicate :

intro ("NOVELL ") is then executed and displays the different modules of this network, such as Description, Requirements, Troubleshooting and Installation. Again the user has to define which module he is interested in . 

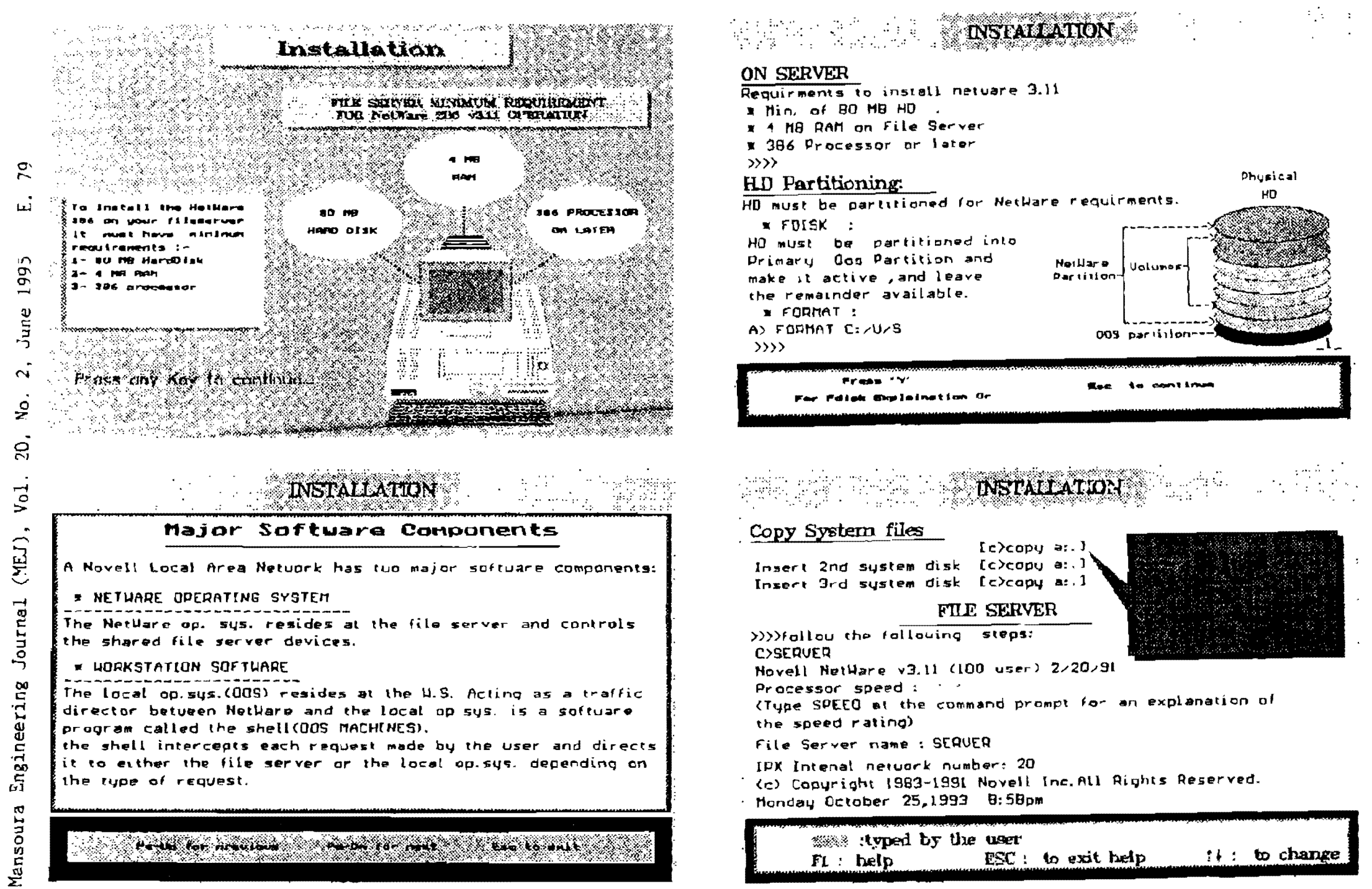

Figure (4) Novell Software and Hardware Installation 
E. 80 Dr. AIDA O. ABD EL-GAWAD
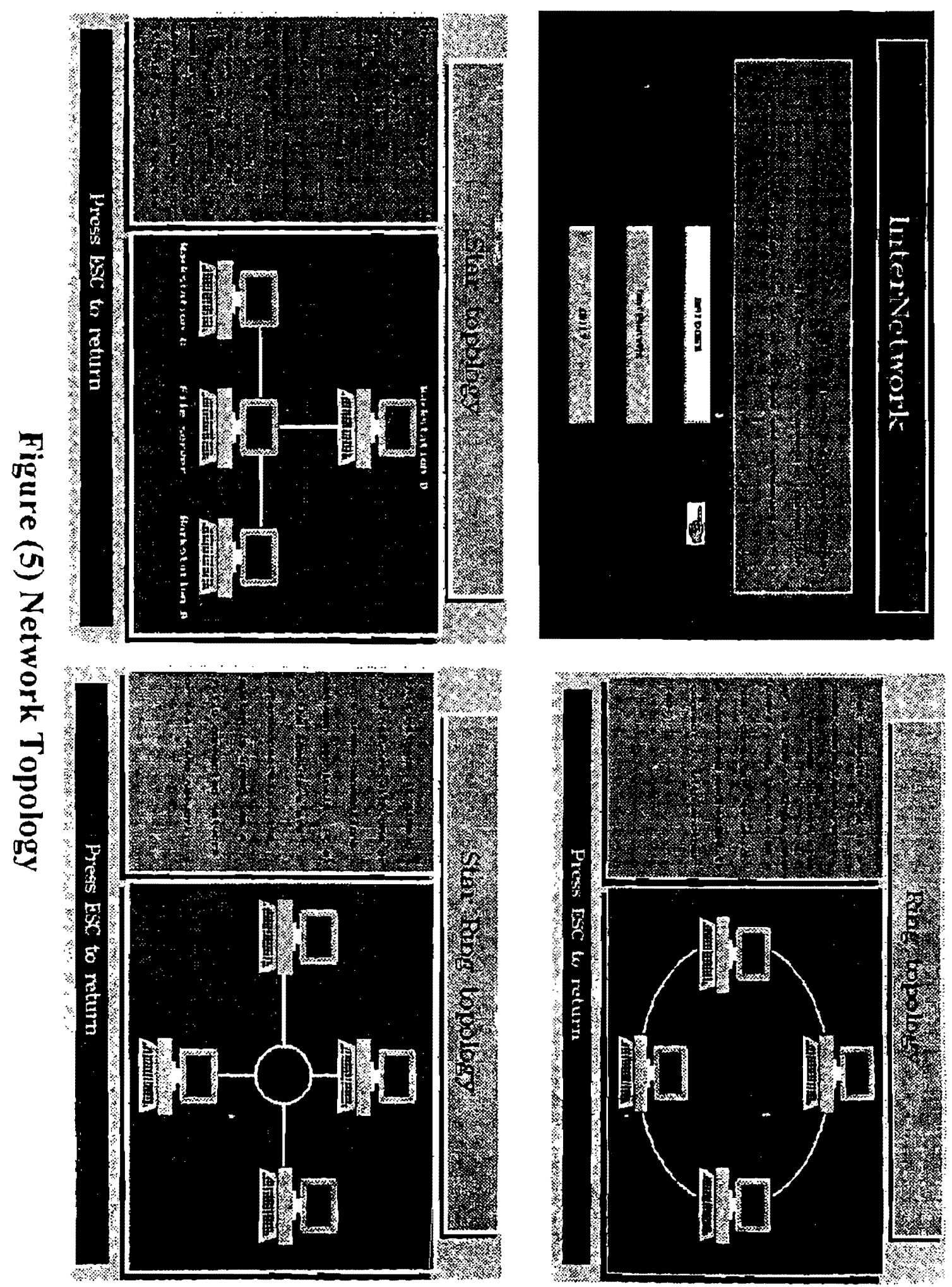
Mansoura Engineering Journal (MEJ), Vol. 20, No. 2, June 1995 E. 81
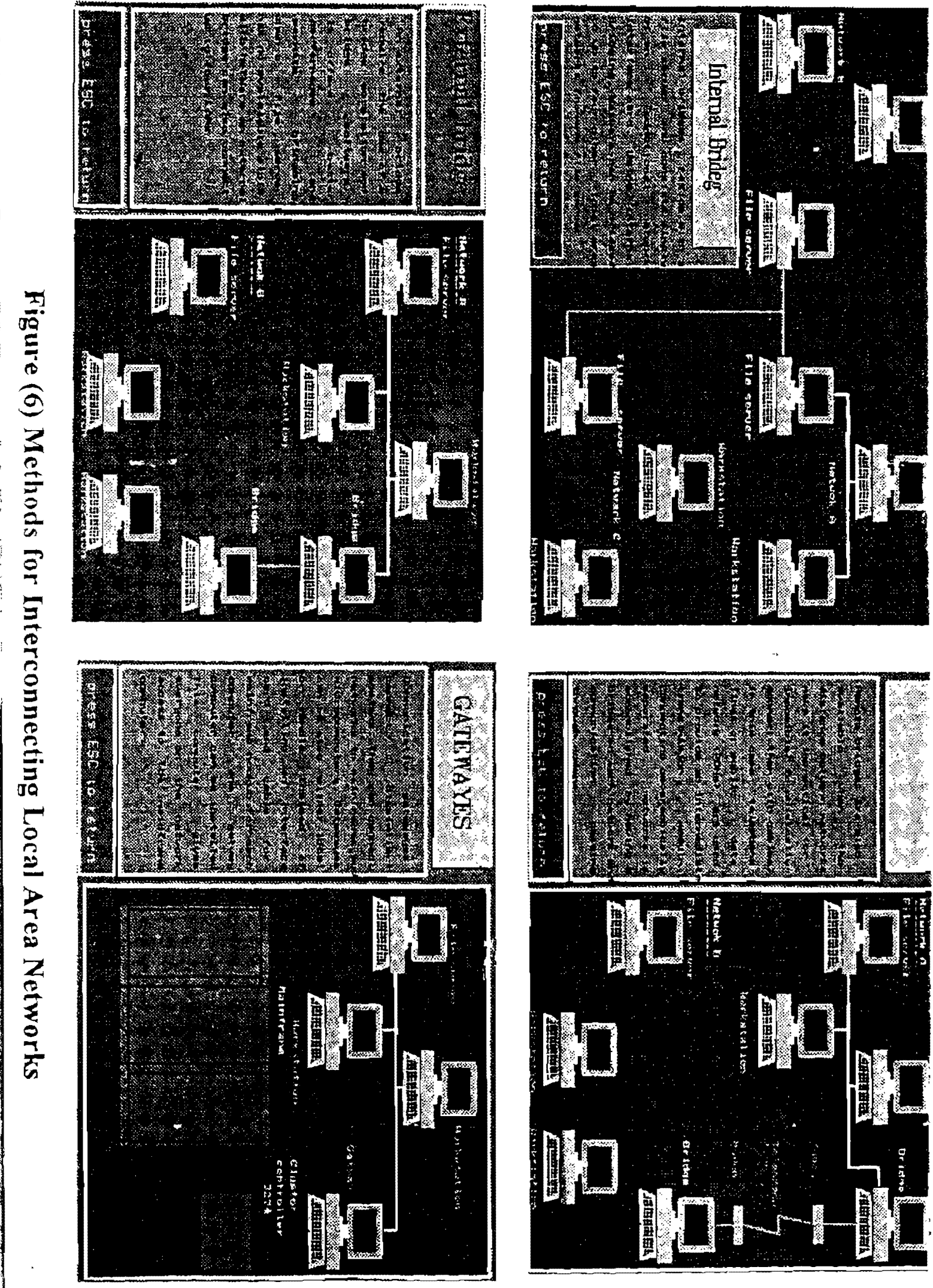

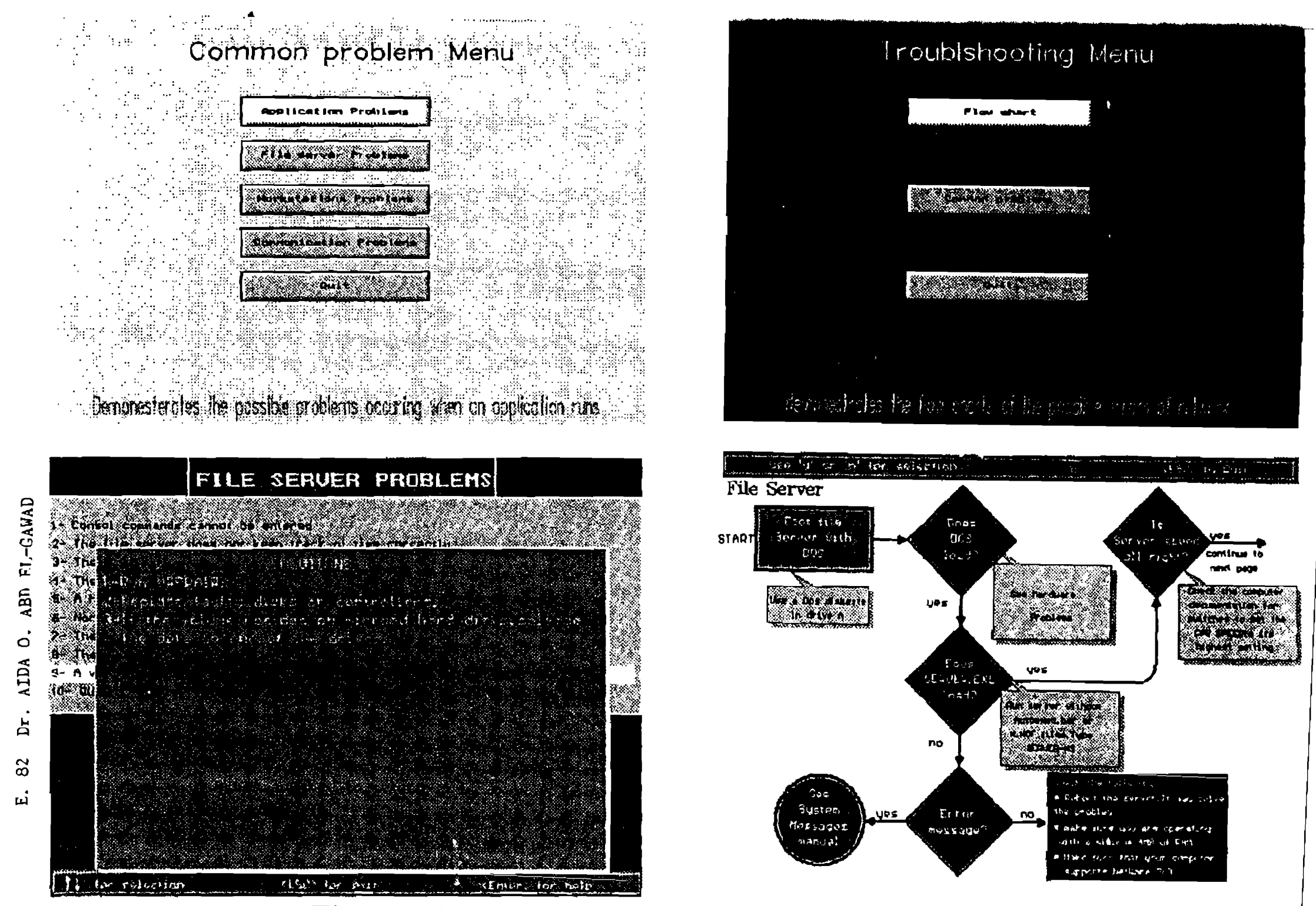

Figure (7) Procedure for Novell Network Diagnosis 
EX.

If we select the Troubleshooting module, then it execute the predicate:

intro ("Troubleshooting ") is then executed and then displays the different parts of this module, such as:

Communication, Workstation, File server and Application problems.

Figure(7) show the designed procedure for novell network diagnoses.

\section{Facts :}

The facts of the knowledge - base are designed in the form :

computer network (novell, pclan, vax),

novell (description, requirements, troublshooting, installation),

description (topology, network basic parts),

requirements (hardware, software)

troubleshooting ( communication, workstation, file server, application

problems),

installation (disk options, volume options, system options),

topology (bus, star, ring, star-ring),

network basic parts (file server, workstation),

hardware (processor, hard disk, ram),

software (file server, workstation).

\section{Explanation facility:} screens.

Explanation facilities are implemented through a very pretty helpful

\section{Conclusion:}

In this paper an expert networking system is introduced. The system is capable of selecting the proper networking type according to the user requirements, accurate and secured data transfer, and high performance multi-processors. Also the system has an excellent methodology for fault diagnoses of networks and introduces a very simple procedure for non expert 
E. 84 Dr. AIUA O. ABD EL-GAWAD

user to set the network parameters and guide him in organizing, and managing the network. The system is easy to maintain, having fast response, and user friendly.

\section{References:}

1- "Computer Network"

Andrew S Tanenbaum, Prentice-Hall 1989

2- "Data Communications, Computer Network and OSI"

Fred Halsall, Addison-Wasley 1988

3- "Local Area Network Architectures"

David Hutchison, Addison-Wasley 1988

4- "Telecommunication Networks:Protocols, Modeling and Analysis" Mischa Schertz, Addison-Wesley 1988

5- "Artificial Intelligence and The Design of Expert Systems"

G. F. Luger and W. A. Stubble field, The Benjanin / Cummings

Publishing Company, inc. , 1990.

6- D. L. Smith , " the ACT : Automatic Computer Troubleshooting project ", 22nd southeastern symp. On system theory, IEEE computer soc. press, pp 383-293, 1990

7- "Local Network Performance"

William Stallings , February 1984 - vol 22 , no. 2 ,IEEE Communication

Magazine.

\section{APPENDIX A}

Computer program for the module of file server troubleshooting. 


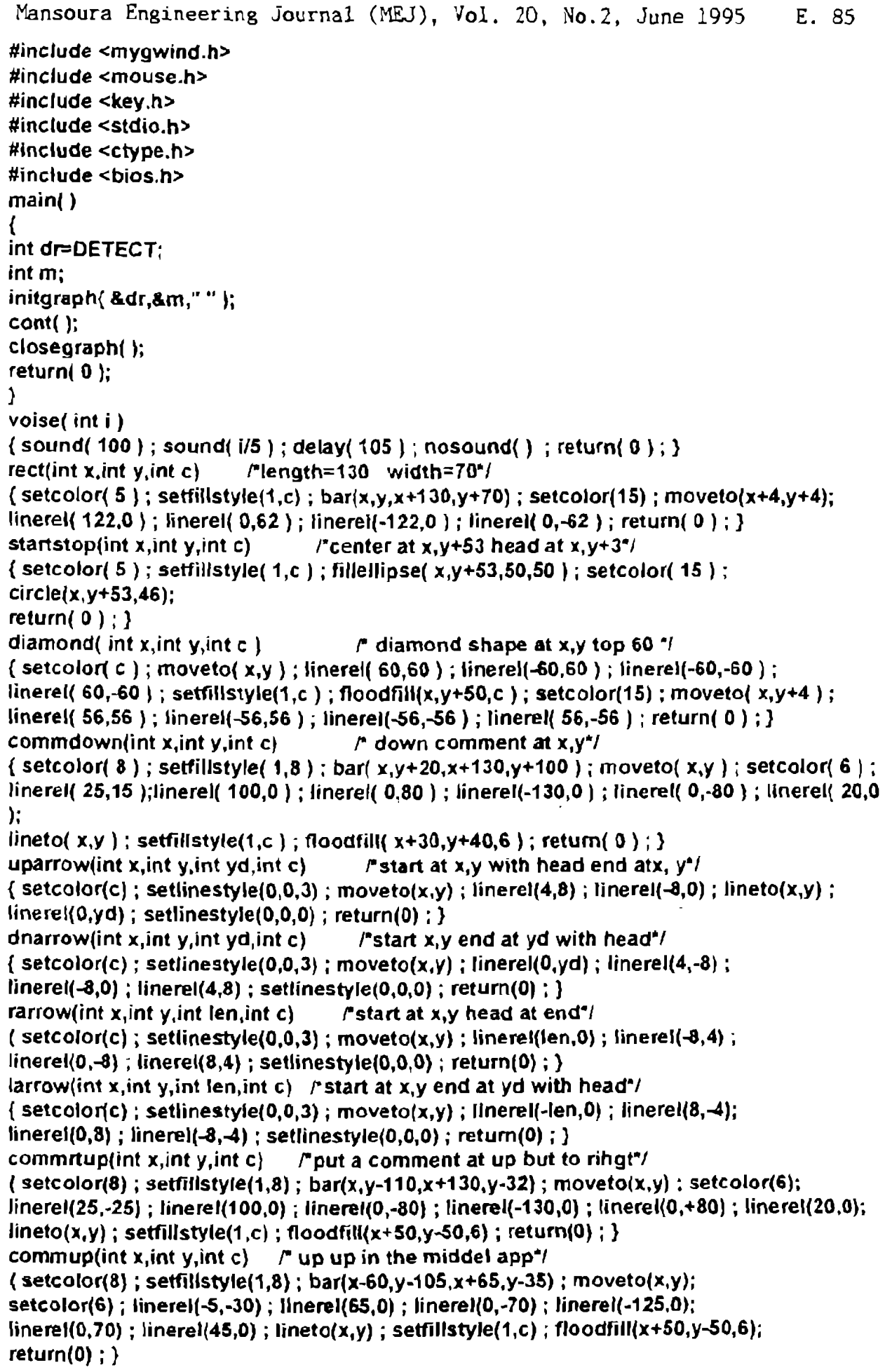


E. 86 Dr. AIDA O. ABD EL-GAWAD

upleft(int $x$,int $y$,int $c$ )

\{ setcolor(8); setfillstyle $(1,8) ; \operatorname{bar}(x-110, y-110, x+20, y-32)$; setcolor $(6)$;

moveto $(x, y) ;$ linerel $(-5,-25)$; linerel $(20,0) ;$ linere $(0,-80)$; linere $(-130,0)$;

linerel $(0,80)$; linerel $(97,0)$; lineto $(x, y)$; setfilstyle $(1, c)$; foodfill $(x-50, y-50,6)$; retum(0) ; \}

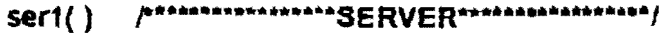

( wind $(1,1,639,479,15,4,0,0) ;$ trst $\}$ ) ; settextstyle $(1,0,2)$; setcolor $(0)$; outtextxy(22,39," File Server") ; graphdefaults( )

$\operatorname{rect}(40,60.1) ; a 11()$;

rarrow $(170,95,35,1)$;

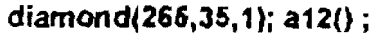

dnarrow $(265,155,30,1)$;

diamond $(265,185,1) ;$ a13) :

dnarrow $(265,305,30,1)$;

diamond $(265,335,1) ;$ a14 $)$;

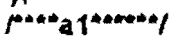

uparrow $(480,155,70,1)$; larrow $(205,395,40,1)$;

startstop $(115,342,1) ; \mathbf{a} 15()$;

diamond $(480,35,1) ;$ a $16(1)$;

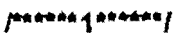

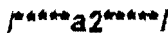

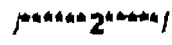

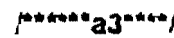

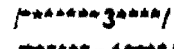

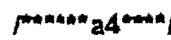

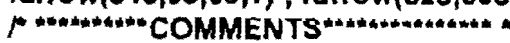

commdown $(305,105,7)$; commdown $(510,125,7) ;$ commdown $(315,240,7)$

commdowns $(80,130,7)$; yesandno 1() ; comser 1() ; contser $(1 ;$ return $(0) ;\}$

contsert()

\{charkey:

do

l

voise $(1000) ; \operatorname{rect}(40,60,1) ; a 11()$;

$\operatorname{delay}(250) ; \operatorname{rect}(40,60,5) ; a 11() ;$

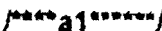

$\operatorname{delay}(250)$; ]

while(bioskey $(1)==0)$;

key $=\operatorname{getch}()$;

while(1) f

switch(key)t

case ESC : retum(0):

default : goto dia;

break; ] \}

dia:

rarrow $(170,95,35,5)$;

dia1: do

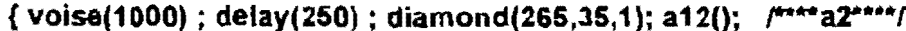

delay(250); diamond $(265,35,5) ;$ a12( $) ;\}$

while(bioske $y(1)==0)$; key=getch () ;

while(1) \{ switch(key)

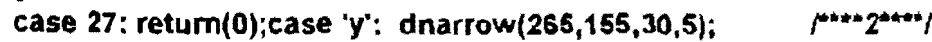

dia2:

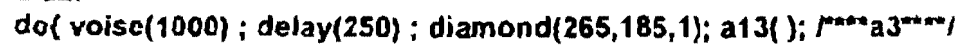

delay (250) ; diamond $\{265,185,5) ;$ a13(): $\}$

masomel

while(bioskey $(1)=0)$; key=bioskey $(0)$

while(1)

\{ swinch(key)

( case ESC: return(0);case ' $y$ ' : setcolor(5) ; setlinestyle $(0,0,3)$;

line $(325,245,460,245) ; \operatorname{arc}(460,225,270,360,20)$; uparrow $(480,155,70,5)$; 
( case ESC: return(0);case ' $y$ ' ; setcolor(5) i setl...estyle $(0,0,3)$;

line $(325,245,460,245) ; \operatorname{arc}(460,225,270,360,20)$; upar: $\cdots(480,155,70,5)$;

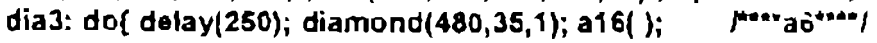

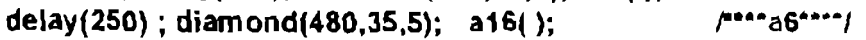

voise $(1000) ;\}$

while(bioskey $(1)==0$ );

key $=\operatorname{getch}($ ):

while(1)

\{ switch(key)

\{ case 'y': rarrow $(540,95,60,5)$; delay $(400)$; ser2( ); return(0) ;

case ESC: retum(0); default: goto dia 3 ; \} \}

break ; case ' $n$ ' : dnarrow $(265,305,30,5)$; pa**ant

dia4: do $\left\{\right.$ delay $(250) ;$ diamond $(265,335,1) ; a 14() ;$ po*a $\left.a 4^{* * * *}\right\}$

delay(250); diamond(265,335,5); a14(); ;

voise $(1000)$; ) while(bioskey(1)==0); key=getch( );

while(1)

\{ switch(key)

\{ case 'y': larrow(205,395,40,5); startstop(115,342,5);a15();return(0);

case ' $n$ ': rarrow $(325,395,35,5)$; wind $(360,340,560,460,10,0,0,0)$; setcolor $(0)$;

settextstyle $(2,0,4)$; settextjustify $(0,2)$;

outtextxy(370,347,"Check the following:"); setcolor(15);

outtextxy(370,363,"* Reboot the server.lt may solve ");

outtextxy(370,378,"the prot em");

outtextxy $(370,395, "$ "* make sure you are operating "

outtextxy $(370,410, "$ with a minimum $4 M B$ of RAM");

outtextxy $(370,427, \cdots$ Make sure that your computer "li

outtextxy $\left(370,442, "\right.$ supports NetWare $\left.368^{\prime \prime}\right)$; return(0);

case ESC: return( 0 ); default : goto dia 4 ; \}) return(0);

defauft: goto dia 2 ; break ; \} break ; )

goto dia1; break; \}) return $(0)$ i)

yesandno1()

( settextstyle $(2,0,5)$; setcolor $(0)$; outtextxy $(240,160$,"yes");

outtextxy(240,310,"no"); outtextxy(350,230,"yes");

outtextxy $(188,385$, "yes") ; outtextxy $(340,385, " n o ")$;

outtextxy(550,85,"yes"); settextstyle $(2,0,4)$;

outtextxy(565,105,"continue to"); outtextxy(565,120,"next page");return(0); \}

comser1()

\{ settextstyle $(2,0,4)$; setcolor $(0)$;

outtextxy(110,16C, "Use a Dos diskette"); outtextxy(110,175,"in drive $A ")$;

outtextxy(340,150," See hardware "); ;

outiextxy $(340,175, " \quad$ Problems"):

outtextxy(565,145," Check the computer") ;

outtextxy(565,160,"documentation for");

outtextxy(565,175," switches to set the ");

outtextxy(565,190,"CPU SPEEDto its"):

outtextxy $(565,205$,"highest setting")i

outtextxy $(365,270$," Run server without");

outtextxy(365,285," Autoexec, bar or");

outtextxy(365,300," ".NCF files. Type");

outtextxy(365,315,"SERVER-NS");

setcolor(13) ; settextjustify $(0,2)$;

outtextxy(370,347,"Check the following:"); setcolor(15);

outtextxy $(370,363$," Reboot the server.ft may solve ");

outtextxy(370,378,"the problem"); 
E. 88 Dr. AIDA 0 . ABD EL-GAWAD

outtextxy(370,395," make sure you are operating ");

outtextxy $(370,410$," with a minimum $4 M B$ of RAM");

outtextxy $(370,427, "$ " Make sure that your computer ");

outtextxy(370,442," supports NetWare 368");retum(0); \}

a11()

( settextstyle $(2,0,5)$; settextjustify $(1,1)$; setcolor(0);moveto $(21,95)$;

outtex("START"); setcolor(15);moveto(105,75); outtext("Boot file");

moveto(105,92); outtext("Server with");

moveto(105,109); outtext("DOS"); return(0); \}

a12()

( moveto(265,75); outtext("Does"); moveto(265,92); outtext("DOS");

moveto(265,109);outtext("load?"); return(0) ; \}

a13()

( moveto(265,225);outtext("Does"):

moveto(265,242); outtext("SERVER.EXE");

moveto(265,259); outtext("load?"); retum(0); )

a14()

( moveto(265,385); outtext("Error");

moveto(265,402);outtext("message?"); return(0); )

a15()

\{ moveto $(115,365)$; Outtext("See");

moveto(115,382); outtext("System");

moveto(115,399); outtext("Messages");

moveto(115,416);outtext("manual"); return(0); \}

a16()

( moveto(480,75);outtext("Is");

moveto $(480,92)$; outtext("Server speed");

moveto(480,109);outtext("all right?"); return(0); \}

ser2()

(wind $(1,1,639,479,15,4,0,0)$; trst(); graphdefaults( );

rarrow $(170,95,35,1)$;

diamond $(400,35,1)$; a22();

$|* 1 *|$

dnarrow $(400,155,30,1)$;

$m a 2 \%$

diamond $(400,185,1) ;$ a23 () :

$m 2^{n} \mid$

dnarrow $(400,305,30,1)$;

diamond(400,335,1); a24();

ras\%

commrtup $(445,390,7)$; rarrow $(460,95,120,1)$;rarrow $(100,95,40,1)$;

diamond(200,35,1); a21();

rarrow $(260,95,80,1)$; larrow $(340,245,80,1)$;

startstop $(210,192,1) ;$ a 25() ;

ra1"I

setlinestyle $(0,0,3)$; setcolor (1); line $(400,455,400,465)$;

larrow $(400,465,90,1)$; larrow $(340,395,30,1)$; setfillstyle(1,1);

wind $(40,345,310,410,1,0,0,0)$;

wind $(40,415,310,470,1,0,0,0)$;

setcolor(1):

/"up wind load diskset'I

line $(200,155,200,180)$; larrow $(200,180,20,1)$; wind $(40,145,180,195,1,0,0,0)$

yesandno2( ); comser2( ); Mwind to enter numbar"/

contser2( ); return(0);

contser2()

(char key; rarrow $(100,95,40,5)$;

dia1: do

diamond $(200,35,1)$; a21();

delay (250); diamond(200,35,5); a21( );

delay (250); voise(1000); \}

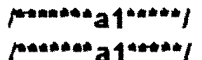


Mansoura Engineering Journal (MEJ), Vol. 20, No. 2, June 1995 E. 89

while(bioskey $(1)==0$ ):

key=getch();

while(1) \{ Switch(key) ( case 'y': rarrow $(260,95,80,5)$;

dia2: do voise(1000); diamond(400,35,1); a22(); $\mathrm{Fa2* \prime}$

delay(250); diamond(400,35,5); a22(): Paz"f

delay $(250)$; J while(bioskey $(1)==0)$;

key=getch ();

while(1)

(switch(key) \&

case ' $y$ ': rarrow $(460,95,120,5)$; delay $(400)$;

ser3( ); return(0); case ' $n$ ': dnarrow $(400,155,30,5) ; M 2 " 1$

dia 3: do voise $(1000)$; diamond $(400,185,1) ; a 23() ; \quad M^{* a}{ }^{*} /$

delay (250); iamond $(400,185,5) ; a 23() ; \quad \mathrm{ra3} \%$

delay (250); ] while(bioskey(1)==0); $\operatorname{key}=\operatorname{getch}()$;

while(1)

\{ switch(key) ( case 'y': larrow $(340,245,80,5)$;

startstop (210,192,5); a25();

$\mid * a 5^{*} /$

return(0); case 'n': dnarrow $(400,305,30,5)$;

dia4: do voise $(1000)$;

diamond $(400,335,1)$; a24( );

delay (250);

diamond $(400,335,5) ; 224() ; \quad \mu a 4$

delay(250):

] while(tioskey $(1)==0$ );

key=getch ();

while(1)

\{ switch(key)

case ' $y$ ':

larrow $(340,395,30,5)$

wind $(40,345,310,410,10,0,0,0) ;$ mup wind load diskset*'

settextstyle $(2,0,4)$; setcolor $(0)$; settextjustify $(0,2)$;

outtextxy $(45,350, "$ Load the DISKSET to check the Hard Disk"):

outtextxy $(45,365$," and controller configuration .");

outtextxy $(45,380$,"Make sure each Hard Disk is assigned to " $)$;

outtextxy $(45,395$," the correct controlier number.");retum(0);

case ' $n$ ':

settinestyle $(0,0,3) ;$ setcolor $(5)$; line $(400,455,400,465)$; larrow $(400,465,90,5)$;

wind $(40,415,310,470,10,0,0,0)$;

settextstyle $(2,0,4)$;

setcolor $(15)$;

settextjustify $(0,2)$;

outtextxy(45,420,"Select the driver settings to match the "):

outtextxy $(45,435$, "hardware settings. or set the disk ");

outtextxy $(45,450$,"coprocessor board to match the driver." );return(0);

case ESC: return(0);default:goto dia4;\})

case ESC: return(0); default:goto dia3;\} Jreturn(0);

case ESC: retum(0); default:goto dia2; \}]

case ' $n$ ': setcolor(5);

"down wind"

larrow $(200,180,20,5)$; wind $(40,145,180,195,10,0,0,0) ;$ settextstyle $\{2,0,4\}$;

setcolor(15); settextjustify $(0,2)$; outte xtxy $(47,157$, "Enter a number that is" $)$;

outtextxy $(47,172, "$ not used by any server"l; retum(0); case ESC: return(0);

default: goto dia1; \} ] rotum(0); \}

yesandno2()

( settextstyle $(2,0,5)$; setcolor(0); outtextxy(270,85,"yes"); 


\section{E. 90 Dr. AIDA O. ABD EL-GAWAD}

outtextxy(470,85,"yes"); outtextxy(370,320,"no"); outtextxy(370,170,"no"); outtextxy(370,455,"no"); outtextxy(210,165,"no"); outtextxy(327,385,"yes"); outtextxy $(310,235$,"yes"); settextstyle $(2,0,4)$; outtextxy(565,105, "continue to"); outtextxy(565,120,"next page"): outtextxy(70,85,"from previous"): outtextxy $(80,100$, "page" $)$; return(0); \}

comser2()

( settextstyle $(2,0,4)$; setcolor(15); settextjustify(0,2);

outtextxy(47,157,"Enter a number that is");

outtextxy $(47,172, "$ not used by any server"); setcolor(0);

outtextxy $(450,300$,"Do the loaded driver");

outtextxy(450,315,"settings match that "):

outtextxy $(450,330$,"for the network disk ");

outtextxy(450,345,"coprocessor boand"); setcolor(15);

outtextxy(45,350,"Load the DISKSET to check the Hard Disk");

outtextxy $(45,365$,"and controller configuration .");

outtextxy $(45,380, "$ Make sure each Hard Disk is assigned to ");

outtextxy(45,395, "the correct controller number,"):

outtextxy $(45,420$,"Select the driver settings to match the ");

outtextxy $(45,435$, "hardware settings. or set the disk ");

outtextxy $(45,450, "$ coprocessor board to match the driver. "); return(0);

a21()

( settextstyle $(2,0,4)$; settextjustify(1,1); moveto(200,70);outtext("Is the");

moveto(200,85); outtext("intemal net-");

moveto(200,100); outtext("work number");

moveto(200,115); outtext("unique?"); retum(0); \}

a22()

(settextstyle(2,0,5); settextjustify(1,1]; moveto(400,75); outtext("Does"); moveto(400,92); outtext("disk driver"); moveto(400, 109);outtext("load?"); return(0); \}

a23()

( settextstyle $(2,0,5)$; settextjustify(1,1); moveto(400,235); outtext("Error"); moveto(400,252);outtext("message?"); retum(0); \}

a24()

( settextstyle(2,0,5); settextjustify(1,1); moveto(400,375);outtext("Do"); moveto(400,392); outtext("setting"); moveto(400,409);outtext("match"); retum(0); ) a25()

\{ settextstyle(2,0,5); settextjustify(1,1); moveto(210,220); outtext("See");

moveto(210,235); outtext("System"); moveto(210,250); outtext("Messages"); moveto(210,265); outtext("manual"); retum(0); \} 